\title{
Mida R, Zager EL (Eds), Surgery of peripheral nerves. A case-based approach
}

\author{
Thieme Medical Publishers, New York, 2008. ISBN 978-0-86577-860-3
}

\author{
Ulrich Sure • Ann-Kathrin Uerschels
}

Received: 6 January 2010 / Accepted: 6 January 2010 / Published online: 4 February 2010

(C) Springer-Verlag 2010

Nowadays, the surgery of peripheral nerves is not practiced any longer in the vast majority of academic neurosurgical institutions. Residents trained in these departments usually do not accumulate any experience in this important field of neurosurgery. Therefore, it is of an utmost importance that some expert neurosurgeons claim the field of peripheral nerves in order to avoid other subspecialties to take it over.

In this respect, Mihda and Sager contribute an important publication to the neurosurgical literature. The publication style as a case-based book is familiar to all of us through our training and daily clinical conferences. The authors dispose the recently more and more evolving topic of peripheral nerve surgery into four sections.

In the first section, selected well-known authors address the entire variety of brachial plexus injuries and entrapment syndromes. There are chapters to all variations of these topics including "gunshot wounds to the brachial plexus" and "fascicular nerve transfers to restore elbow flexion". The second section covers all possible variations of upper extremity peripheral nerve injuries and entrapments with nice contributions to endoscopic techniques and rare occasions such as the "anterior interosseous nerve syndrome". The third section comprises the entire information on the lower extremity peripheral nerve injuries and entrapments. The most interesting part of this publication is summarized in the fourth section on "nerve tumors, painful nerve conditions and miscellaneous injuries". Here, the broad variety of tumors in the various possible locations is illustrated and well described. Moreover, sympathectomy for hyperhidrosis and electrical stimulation for painful neuroma are covered by small chapters.

This comprehensive book on peripheral nerve surgery is well written and illustrated. It is highly instructive because it contains numerous nice operative photographs and/or artistic sketches as well as very useful sections on "Pearls" and "Pitfalls". All chapters contain an informative case presentation with the necessary diagnostics, anatomy, clinical evaluations, management options with special reference to the surgical treatment, and outcome data, respectively. Furthermore, a suggested reading part for colleagues who want to refer to the primary literature is provided. Similar to previous publications, unfortunately, color printing is lacking, particularly of intraoperative photographs.

In summary, the present book is highly recommended for colleagues who are interested in the pathology and neurosurgical treatment of the peripheral nervous system.
U. Sure $(\bowtie) \cdot$ A.-K. Uerschels

Klinik für Neurochirurgie, Universitätsklinikum Essen,

Essen, Germany

e-mail: ulrich.sure@uni-due.de 